Thorax (1956), 11, 223.

\title{
THE DIRECT INTERPRETATION OF THE FAST VITAL CAPACITY RECORD
}

\author{
BY \\ R. J. SHEPHARD \\ From the R.A.F. Institute of Aviation Medicine, Farnborough, Hants
}

(RECEIVED FOR PUBLICATION JANUARY 30, 1956)

The standard maximum breathing capacity test (Hermannsen, 1933; Gilson and Hugh-Jones, 1949) places a severe strain on a patient who is unwell, and in a diseased lung it may lead to structural damage with exacerbation or spread of infection (Simonin, 1954; Needham, Rogan, and McDonald, 1955). The search for an alternative and less exhausting procedure has concentrated increasingly on the fast vital capacity (F.V.C.) manœuvre. This has been considered largely as a means of predicting maximum breathing capacity (the relevant literature is reviewed by Kennedy, 1953), although there have also been pleas for a more direct interpretation of F.V.C. tracings (Bernstein and Kazantzis, 1954 ; Shephard, 1955).

If F.V.C. tracings are to be interpreted directly, it is necessary to be certain to what extent the form of the tracing is affected by slight variations in the apparatus or the technique adopted by the patient. Instrumental errors may be of considerable importance. In the past, records (sometimes termed fast vital spirograms) have been obtained by means of a spirometer fitted with either a standard brass bell (Kennedy, 1953 ; Needham and others, 1955) or a light-weight bell (Bernstein, 1954); the two methods yield tracings that differ considerably from each other, and neither corresponds very closely with the pattern of flow recorded by the pneumotachograph (Shephard, 1955). The present paper reports an investigation of errors underlying the spirometer methods of measuring F.V.C. and a study of the stability of the various F.V.C. parameters by repeated pneumotachograph measurements on a small group of normal subjects.

\section{METHODS}

The PNeumotachograph.-Details of the pneumotachograph have been given in previous reports (Shephard, 1955 ; 1956). This apparatus has a $95 \%$ response time of under 100 milliseconds, and the accu- racy is not less than $1 \%$ even with the high rates of gas flow found in very fit subjects.

The SPIROMETER.-A Knipping type spirometer was adapted for electrical recording by bolting a ballbearing mounted potentiometer to the pulley. Careful comparison of pressure tracings obtained from the bell during fast vital capacity manœuvres failed to reveal any significant change following attachment of the potentiometer. A light-weight celluloid bell (Bernstein and Mendel, 1951) was used in some experiments. With this bell, the total weight of the moving parts was reduced to $612 \mathrm{~g}$., and a maximum unbalance of 75 g. occurred with full immersion.

The SubJects.- The male subjects were fairly representative of the range of physiques likely to be encountered in healthy young men. On the other hand the female subjects represent only the two extremes of physical development, one (V.G.) being a championship swimmer, the other (V. B.) being small, with poor musculature. The physical characteristics of all except V.G. have been summarized in a previous report (Shephard, 1955). She had a height of $5 \mathrm{ft} .6 \frac{1}{2}$ in. (169 cm.), a weight of $142 \mathrm{lb}$. (64.2 kg.), and a body surface of 1.74 sq. $\mathrm{m}$.

\section{Errors Associated with Spirometer Method OF RECORDING FAST VITAL CAPACITY}

Simultaneous PNeumotachograph and SPIRoMETER RECORDS.-In order to determine whether the pattern of gas flow during a fast vital capacity manœuvre is distorted by the physical characteristics of the spirometer system, a pneumotachograph was inserted in the tubing leading to the spirometer and gas flows recorded during movement of the spirometer bell. Tracings obtained in this way were compared with (a) previously obtained normal pneumotachograph tracings for the same subject and $(b)$ the simultaneously recorded spirometer volume.

Comparison with normal pneumotachograph tracings (Table I) shows that there is little change in the total time taken over the fast vital capacity 
TABLE I

PATTERN OF GAS FLOW DURING FAST VITAL CAPACITY MANEUVRE AND EFFECT OF INCREASING INITIAL RESISTANCE TO GAS FLOW BY USE OF SPIROMETER BELL

\begin{tabular}{|c|c|c|c|c|c|c|}
\hline & \multicolumn{2}{|c|}{$\begin{array}{l}\text { Pneumotacho- } \\
\text { graph } \\
\text { Alone }\end{array}$} & \multicolumn{2}{|c|}{$\begin{array}{l}\text { Addition of } \\
\text { Brass Spirometer } \\
\text { Bell and Tubing }\end{array}$} & \multicolumn{2}{|c|}{$\begin{array}{l}\text { Addition of Cellu } \\
\text { loid Spirometer } \\
\text { Bell and Tubing }\end{array}$} \\
\hline & I.F.V.C. & E.F.V.C & I.F.V.C. & E.F.V.C. & I.F.V.C. & E.F.V.C. \\
\hline $\begin{array}{l}\text { Time to } \\
\text { peak gas }\end{array}$ & & & & & & \\
\hline $\begin{array}{l}\text { flow (sec.) } \\
\text { Total time }\end{array}$ & 0.58 & 0.51 & 0.46 & $0 \cdot 20$ & 0.38 & 0.21 \\
\hline $\begin{array}{l}\text { (sec.) .. } \\
\text { Peak gas }\end{array}$ & $2 \cdot 14$ & 2.51 & $2 \cdot 00$ & 2.94 & 1.98 & 3.43 \\
\hline $\begin{array}{l}\text { low } \\
\text { min. } \\
\text { B.T.P.S.) }\end{array}$ & 169 & 291 & 317 & 448 & 226 & 450 \\
\hline (m.T.P.S.) & 3,230 & 3,810 & 3,450 & 3,700 & 2,560 & 3,470 \\
\hline
\end{tabular}

manœuvre, but a rather earlier and higher peak flow is observed when the subject is connected to a spirometer, this effect being more marked with the brass than with the lighter celluloid bell.

The simultaneous records show a good correspondence between the pneumotachograph indication of gas flow and the changes of spirometer volume, the main weakness of the volume record being its failure to indicate the precise moment when the subject begins the vital capacity manœuvre. This is because the gas flow initially is small, particularly during inspiration. The order of error in the timing of vital capacity measurements can be gauged from observations on a normal subject, although in pathological subjects it may tend to be rather greater. In subject D. P. 16 observations of inspiratory lag gave a value of $167 \pm 61$ milliseconds, and 15 observations of expiratory lag gave a value of $89 \pm 9$ milliseconds.

Lack of balance (up to $90 \mathrm{~g}$. in some
TABLE II

TIMES TO PEAK FLOW (E.F.V.C., SUBJECT D. P.)

$\begin{array}{lllll}\text { Brass bell (fully counterbalanced) } & \ldots & \ldots & \ldots & 0.219 \\ \text { sec. }\end{array}$

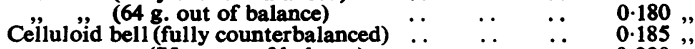

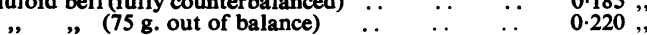

$\mathrm{H}_{2} \mathrm{O}$. As will be seen later, this represents a small fraction of the total pressure required to initiate movement of the bell, and therefore has little effect on the time required to reach peak velocity.

Fluctuations in the F.V.C. ReCORD.-It has been emphasized recently that the form of the F.V.C. tracing is not invariably a smooth curve. Variations in the pattern of gas flow may occur according to the technique of delivery adopted by the subject (Shephard, 1955). However, the spirometer volume record shows other gross fluctuations, and there is good reason to believe that these are introduced by the apparatus (Bernstein and Mendel, 1951). The physical basis of these volume fluctuations may be examined conveniently in terms of the exciting pressure wave and the nature of the resonating system; their effect on the form of the F.V.C. record will then be considered.

The Exciting Pressure Wave.-Bernstein and Mendel (1951) predicted that a pressure would develop in the spirometer bell during the F.V.C. manœuvre on account of the inertia of the moving parts. To determine the form of this pressure wave, diagrams have been plotted on the cathoderay screen showing spirometer pressure in relation to time and to the volume of gas entering or leaving the spirometer.

The records of pressure against time (Fig. 1) show the form that might be expected from the pneumotachograph tracings of flow. During the I.F.V.C. the pressure reaches a maximum value of 40 to $50 \mathrm{~mm} . \mathrm{H}_{2} \mathrm{O}$ in 0.1 to 0.2 second (correlight-weight s p ir o meter systems) might be expected to introduce an error by producing an excessive acceleration of the moving parts. In practice this does not seem of great importance, the various spirometer systems taking similar times to reach peak flow (Table II).

The probable explanation is that lack of balance in the closed system is translated immediately into a change of gas pressure amounting in this case to some $4 \mathrm{~mm}$.

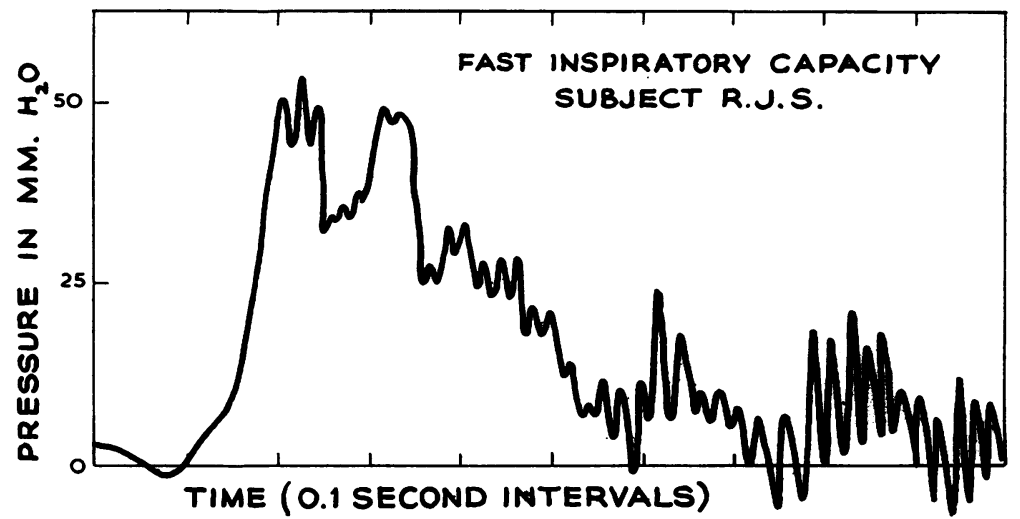

FIG. 1.-Pressure fluctuations in the spirometer during fast vital capacity manœuvre. 
sponding with the period of maximum acceleration of the bell), and has fallen virtually to zero at 0.5 second (corresponding with the point where deceleration of the bell begins). Superimposed on this main pressure wave are a number of smaller rhythmic fluctuations of pressure. The most obvious of these has a medium frequency (c. $600 /$ minute), but there is also a well-defined fluctuation of smaller amplitude and low frequency (c. 60/minute), and in some tracingsapparently when a high velocity is reached-a high frequency component (c. 3 to $3,500 /$ minute).

During expiration a more rapid maximum pressure is attained ( 0.07 to 0.1 second), the pressure achieved is higher $\left(100\right.$ to $\left.110 \mathrm{~mm} . \mathrm{H}_{2} \mathrm{O}\right)$, and the fall to zero pressure is more rapid (often taking as little as 0.2 to 0.3 second). The superimposed rhythmic fluctuations of pressure are similar to those seen during inspiration, although the high frequency component is more consistently present.

When a light-weight spirometer bell is used the timing of the main pressure wave is not substantially altered, but its magnitude is approximately halved (inspiration 20 to $25 \mathrm{~mm}$. $\mathrm{H}_{2} \mathrm{O}$, expiration 45 to $55 \mathrm{~mm}$. $\mathrm{H}_{2} \mathrm{O}$ ). The three frequencies of rhythmic pressure fluctuation are observed as before, and the amplitude of the high and medium frequency components is somewhat increased.

Pressure/volume loops obtained for the brass spirometer bell confirm the above findings. During inspiration a peak pressure of about $40 \mathrm{~mm}$. $\mathrm{H}_{2} \mathrm{O}$ is reached with the movement of a few millilitres of gas; the pressure then falls rather slowly to about $25 \mathrm{~mm}$. $\mathrm{H}_{2} \mathrm{O}$ at a point half-way through inspiration. The second half of the fast inspiration shows a rapid fall of the spirometer pressure towards zero. During expiration a peak pressure of about $100 \mathrm{~mm} . \mathrm{H}_{2} \mathrm{O}$ is reached with virtually no movement of gas. After the expulsion of a few hundred millilitres of gas the pressure falls rapidly, and during the last half of expiration is not greater than $10 \mathrm{~mm} . \mathrm{H}_{2} \mathrm{O}$.

Nature of the Resonating System.-Theoretically, the spirometer is a rather complex resonator. Two oscillating systems of considerable mass and differing natural period (the bell-chain-counterweight system, and the U-loop of the water seal) are connected by a highly elastic system-a gas space containing air or oxygen.

Further, the gas has a variable distribution between subject and apparatus. Two compound pendulums connected by a light spring of variable tension provide a simple physical analogy. A precise mathematical determination of natural frequency is difficult for such a complex system, but

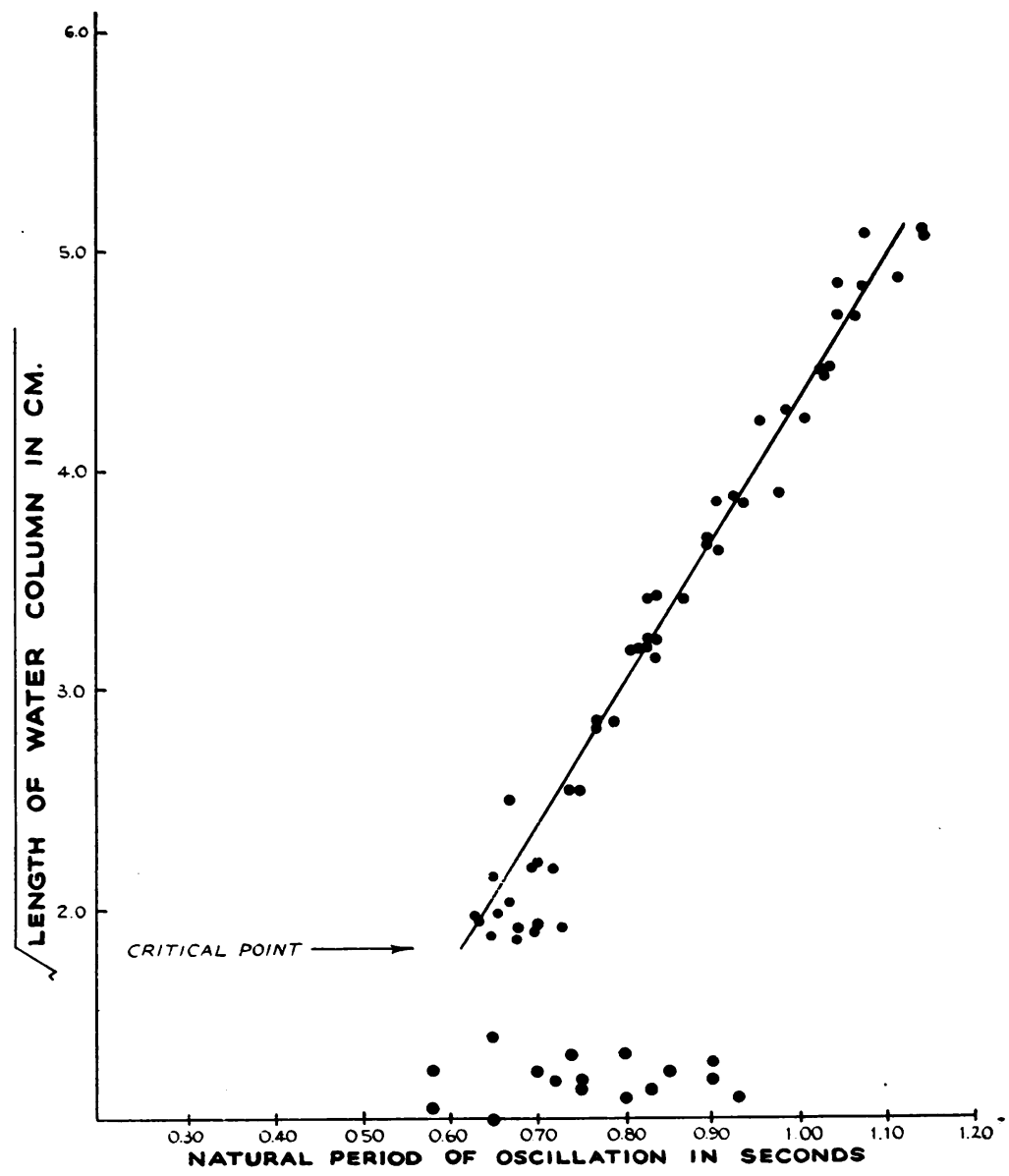

FIG. 2.-Relationship between square root of length of water column and natural period of oscillation of spirometer. (Brass bell, oscillations induced by removal of standard mass.) 
it can readily be seen that the dominant frequency will be determined by the relative masses of the water column and the bell-counterweight systems; where these are of similar mass, interference between the two systems may give rise to "beats."

From the practical point of view, it is obvious that the water column has much the greater mass when the bell is fully immersed, but it is important to be certain whether the water loop continues to act as the dominant resonating system over the useful range of the apparatus. To investigate the natural frequency with differing lengths of water column, oscillations were induced by the sudden removal of a standard mass $(600 \mathrm{~g}$.) from the top of the spirometer bell. There was a close relationship between the period of the oscillations and the square root of the length of the water column (Fig. 2) until the depth of immersion reached a critical value (about $4 \mathrm{~cm}$. for the brass bell). At this point the oscillations became irregular in character; sometimes excessive damping occurred, and sometimes " beats" were observed. It is difficult to make an exact comparison between the mass of the water column and the mass of the bell in this experiment, since the "loop" of water extends for an unknown distance below the edge of the bell. However, if an "end-correction" of $6 \mathrm{~cm}$. - a not unreasonable figure-is assumed, the mass of the two systems would be approximately equal when the bell was immersed to $4 \mathrm{~cm}$. This seems at least a tenable explanation of the unstable frequency observed at this level. Since the mass of the light-weight bell system is only about one-third of that of the brass bell, the critical frequency would be expected to occur with even less immersion of the bell. It is difficult to produce satisfactory oscillations with a water column less than 1 $\mathrm{cm}$. in length, but it can be shown that down to about $1 \mathrm{~cm}$. there is a close linear relationship between the natural period and the square root of the length of the water column. Thus these observations establish two important practical facts-the lowest frequency fluctuations observed with both types of spirometer bell are governed by the length of the water column down to a certain critical point, and this point is not reached unless the bell is overfilled with gas or the water-level is low.

The medium and high frequency vibrations are not of great practical importance, since they are both associated with the use of the spirometer, and the normal recording system-the ink-writing spirometer pen-is not capable of responding to such frequencies. They are both rather rapid to be attributed to the moving parts of the spirometer, and probably represent resonance of the gas spaces in the apparatus and patient. Their amplitude is much increased if the "noise" level is raised by an increased speed of delivery or voluntary modifications in the shape of the subject's airway.

Wave-form during F.V.C. Manœuvre-Details of the wave-form produced by removal of the standard mass are summarized in Fig. 3a. A virtually "square" pressure change of $27 \mathrm{~mm}$. $\mathrm{H}_{2} \mathrm{O}$ gives rise to a chain of vibrations with an initial amplitude of some $200 \mathrm{ml}$. The damping constant (time required for the amplitude to fall to $\mathrm{e}^{-1}$ of a given value) is greater with a long water column (due mainly to the lower frequency), and is also considerably increased with the celluloid bell (probably due to its more highly polished surface). The pattern of damping conforms fairly closely to an exponential curve, suggesting that the viscous component exceeds frictional resistance by at least an order of magnitude.

Do the less regular pressure changes associated with the F.V.C. manœuvre produce similar fluctuations in the spirometer volume record? In order to calculate the wave form during an F.V.C. test, it is necessary to correct for the overall movement of the bell. This may be done if it is assumed that a given half-wave is equal in amplitude to the mean of preceding and succeeding half-waves-an approximation that is correct to about $5 \%$ under the conditions of the experiment. The observed frequency of the oscillations is close to that produced by the "square" pressure wave, but the degree of damping is appreciably greater, particularly during the E.F.V.C. The most striking difference is in the amplitude of vibration. Although the pressure developed during an E.F.V.C. is about four times that produced by removal of the standard mass, the unfavourable shape of the pressure wave results in an oscillation of little greater initial amplitude. The pressure wave produced by the light-weight bell apparently has an even less favourable shape, and the initial amplitude is only about one-quarter of that observed with the brass bell.

The effect of these oscillations on the accuracy of volume recordings must finally be considered. It seems obvious that neither type of spirometer bell is capable of giving an accurate picture of events occurring during the first second, but if only the capacity at one second is being measured the situation is less serious. The error will vary somewhat according to the precise phase of the wave cycle at one second, but is of the order 100 to $120 \mathrm{ml}$. $(2.5$ to $3.0 \%)$ for the brass bell, and 

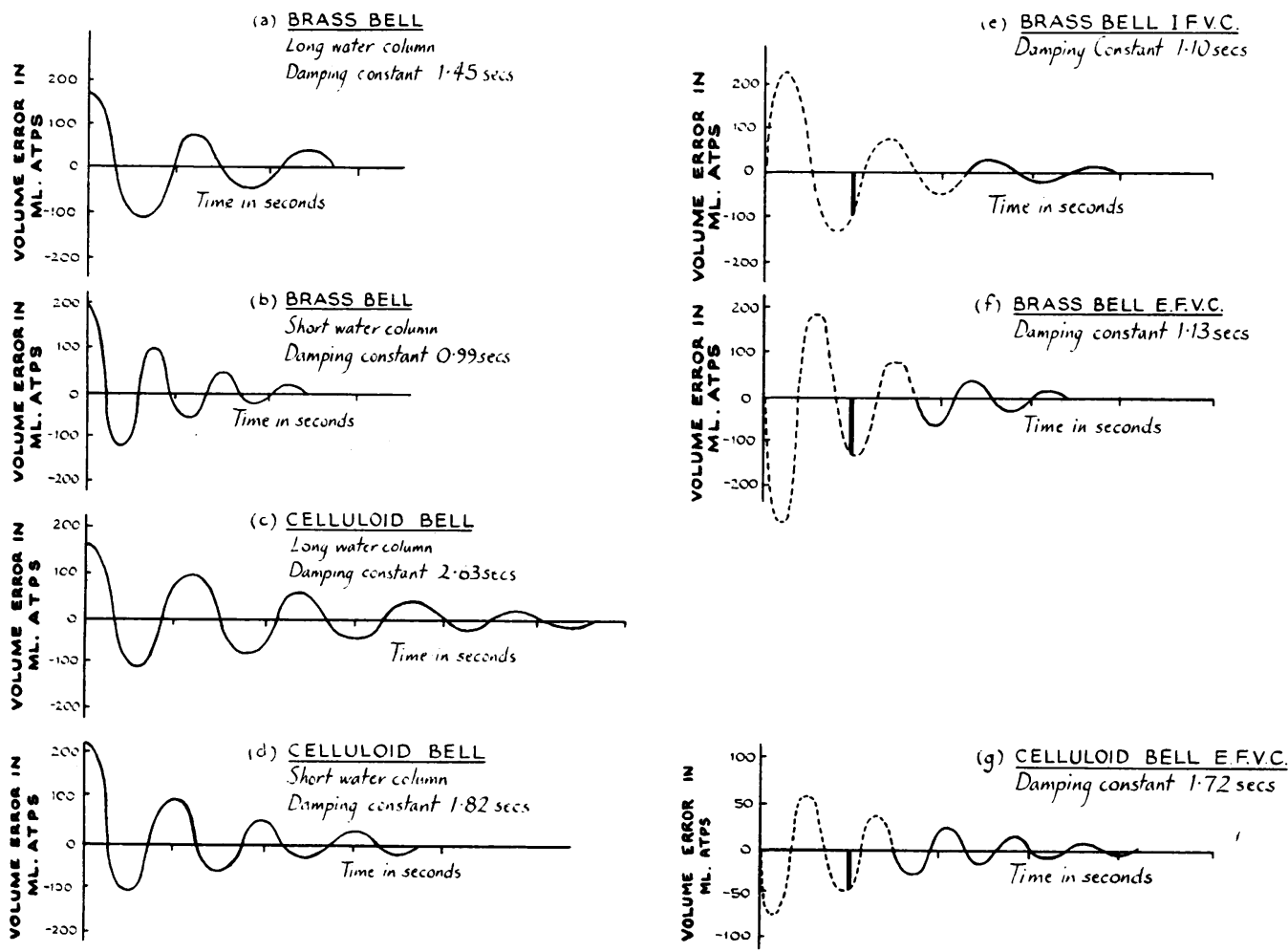

FIG. 3.-Wave form of spirometer oscillations. (a)-(d) Oscillations induced by "square" pressure change of $27 \mathrm{~mm}$. $\mathrm{H}_{2} \mathrm{O}$; (e)-(g) reconstructed wave form from actual fast vital capacity tracings. Solid lines indicate waves actually measured.

$40 \mathrm{ml}$. (1\%) for the celluloid bell. Errors of this magnitude may not be of clinical importance, but their existence must be remembered when comparing results with those obtained by the pneumotachograph.

\section{The Parameters of Fast Vital Capacity}

When instructed to perform a vital capacity manœuvre as rapidly as possible, subjects vary considerably in the time taken. In normal subjects a "fast inspiratory capacity" may occupy 1.1 to 2.1 seconds, and a "fast expiratory capacity" 2.2 to 4.6 seconds. If patients are being examined the range of times is even wider (Shephard, 1956). It is therefore of some importance to ascertain how a variation in the total time taken over delivery affects other parameters of the fast vital capacity

To investigate this point, two normal subjects delivered nearly 200 expiratory and inspiratory vital capacity samples, taking one to ten seconds over the delivery. Twelve tracings were also obtained from each of six other normal subjects. The following features of the individual pneumotachograph records were noted :

Total Gas Volume Delivered.-The total volume delivered is closely dependent on the time taken over delivery. It may conveniently be expressed as a percentage of the untimed vital capacity measured by the spirometer. There is no significant difference between the percentage values for the two principal subjects, and, to facilitate graphic analysis, results from these two subjects have therefore been pooled (Fig. 4). A consistently larger fraction of the expiratory capacity than of the inspiratory is delivered in a given time, 98 to $100 \%$ of the total capacity being achieved in three seconds. The inspiratory capacity apparently reaches a limiting level in about two and a half seconds, and if longer time is taken may even 


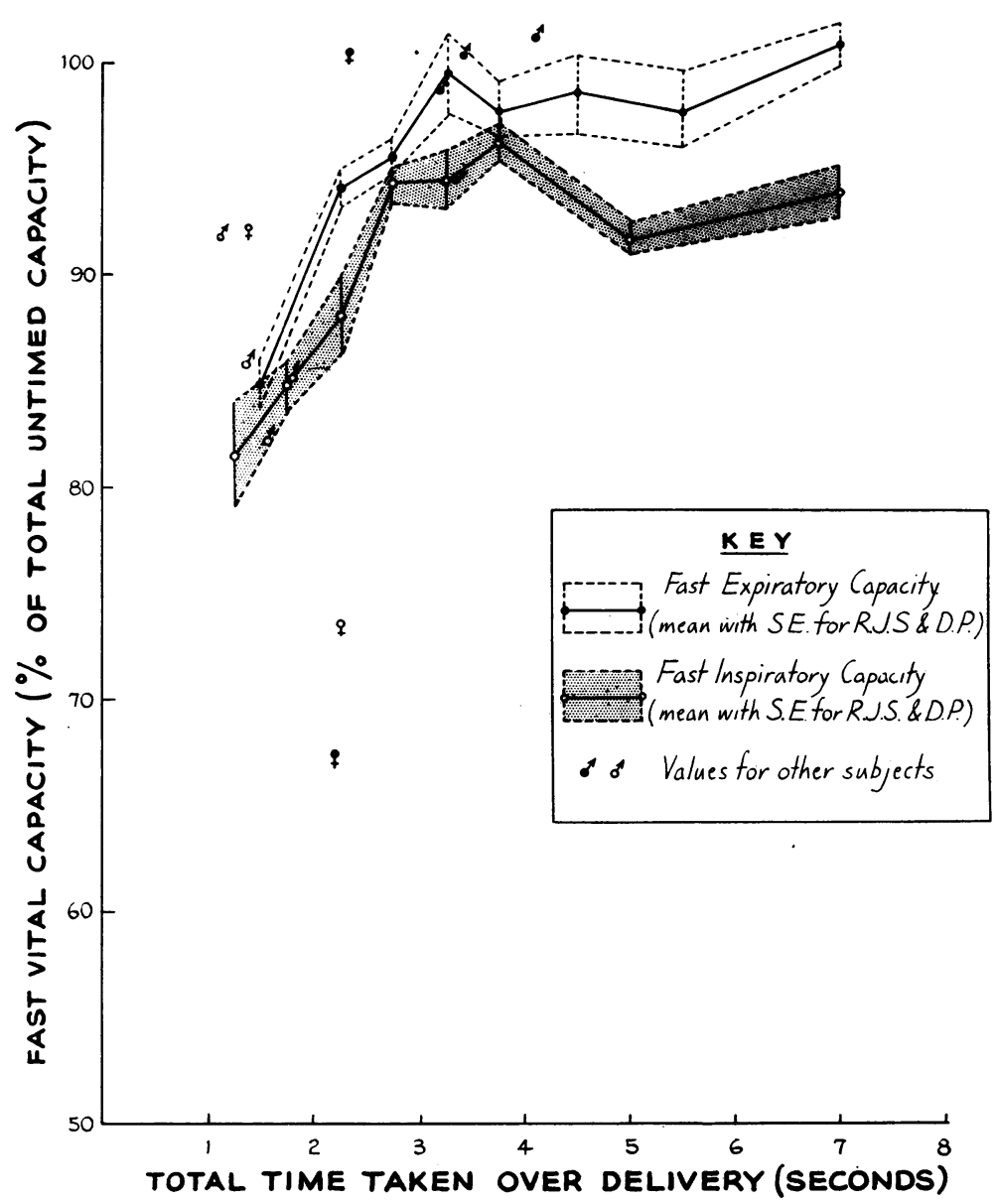

Frg. 4.-Relationship between volume of fast vital capacity and time taken over delivery of sample.

show a slight decrease. It does not rise above about $95 \%$ of the untimed expiratory capacity.

Values for most of the other male subjects fall fairly closely along these curves. One female subject (V. B.) delivers a much smaller fraction of the untimed capacity in a given time, but this is probably related to her build rather than her sex, since the other (V.G) is even somewhat above the average male percentage.

Gas Volume Delivered in One Second.-In order to assess the fast vital capacity most previous authors have selected an arbitrary (usually one second) fraction of a fast spirometer tracing. A corresponding volume can be obtained by the pneumotachograph technique if the area of a onesecond portion of the tracing is measured by a planimeter.

If the total untimed vital capacity is taken as a standard, and the percentage of this standard delivered in one second is plotted against the time taken over delivery (Fig. 5), it can be seen that the "one-second capacity" is not a constant entity. Considering first the pooled results for the two principal subjects, the curve relating the onesecond expiratory capacity to delivery time approximates to a sigmoid form. Over the section 1 to 3 seconds there is virtually no change of capacity, values being mainly in the "normal" range (70 to $80 \%$ of untimed capacity) (Hirdes and van Veen, 1952; Gaensler, 1951 ; Simonin, 1954 ; Drutel and Dechoux, 1952). From 3 to 6 seconds the capacity falls rapidly to some $35 \%$ of the untimed value, but there is little further deterioration in isolated observations where the delivery time has been prolonged even to 12 seconds. With the inspiratory capacity, speed of delivery is even more important. Values in the "normal range" (70 to 
$80 \%$ of untimed capacity) cannot be achieved unless the inspiration is completed within 2 seconds. If longer is taken over inspiration the one-second capacity rapidly falls to about $35 \%$ of the untimed capacity, the course of the curve lying significantly to the left of that for the one-second expiratory capacity. Results for the other male subjects fall closely along these curves. There are again considerable differences between the two female subjects, one showing low values (particularly for expiratory capacity) and the other exceeding the average male values.

The magnitude of the onesecond capacity bears a close relationship to the peak rate of gas flow (Fig. 6). The two principal subjects had to achieve a peak flow of $300 \mathrm{l} / \mathrm{min}$. during expiration and $275 \mathrm{l} . / \mathrm{min}$. during inspiration in order to attain a capacity $70 \%$ of the untimed value. The inspiratory curve lies consistently to the left of expiratory, this difference being statistically significant in its upper part.

The Peak Rate of Gas Flow. - There is a highly significant relationship between the total time taken over delivery of the sample and the peak flow rate achieved (Fig. 7). The behaviour during the expiratory manœuvre can be described quite satisfactorily by means of a linear regression, and there is no evidence that a limiting velocity is being approached even if as little as one second is taken over delivery. The inspiratory values are described less completely by a linear regression, since there is some evidence of a disproportionate change of flow rate over the range 1 to 2 seconds ; however, from 2 to 6 seconds the points again fall fairly closely about a straight line. It will be noted that the expiratory regression lies significantly to the right of the inspiratory $(P<0.001)$, although the slopes of the two lines are very similar.

The Flow Rate at One Second.-The original premise underlying the "one-second capacity" was that the gas flow during the F.V.C. manœuvre remained at or near a maximum for about one second in normal subjects (Hirdes and van Veen,
1952). The validity of this concept has been tested by calculating the flow rate at one second as a percentage of the peak flow in any given tracing. It was found that the one-second velocity varied considerably with the total time taken over delivery of the sample. However, in 85 of 103 expiratory tracings and 80 of 88 inspiratory tracings, the peak velocity had been passed, and most observations with a normal delivery time had a one-second flow rate that was only 40 to $60 \%$ of the peak value.

If the period of measurement was shortened to 0.75 second, as suggested by Kennedy (1953), there was some increase in the number of readings where the peak flow had yet to be reached, but this was not great -19 of 88 inspiratory tracings, and 33 of 103 expiratory tracings. In most tracings the velocity at 0.75 second was only slightly greater than at 1 second, and there was no evidence of a sharp break in the velocity curve at 


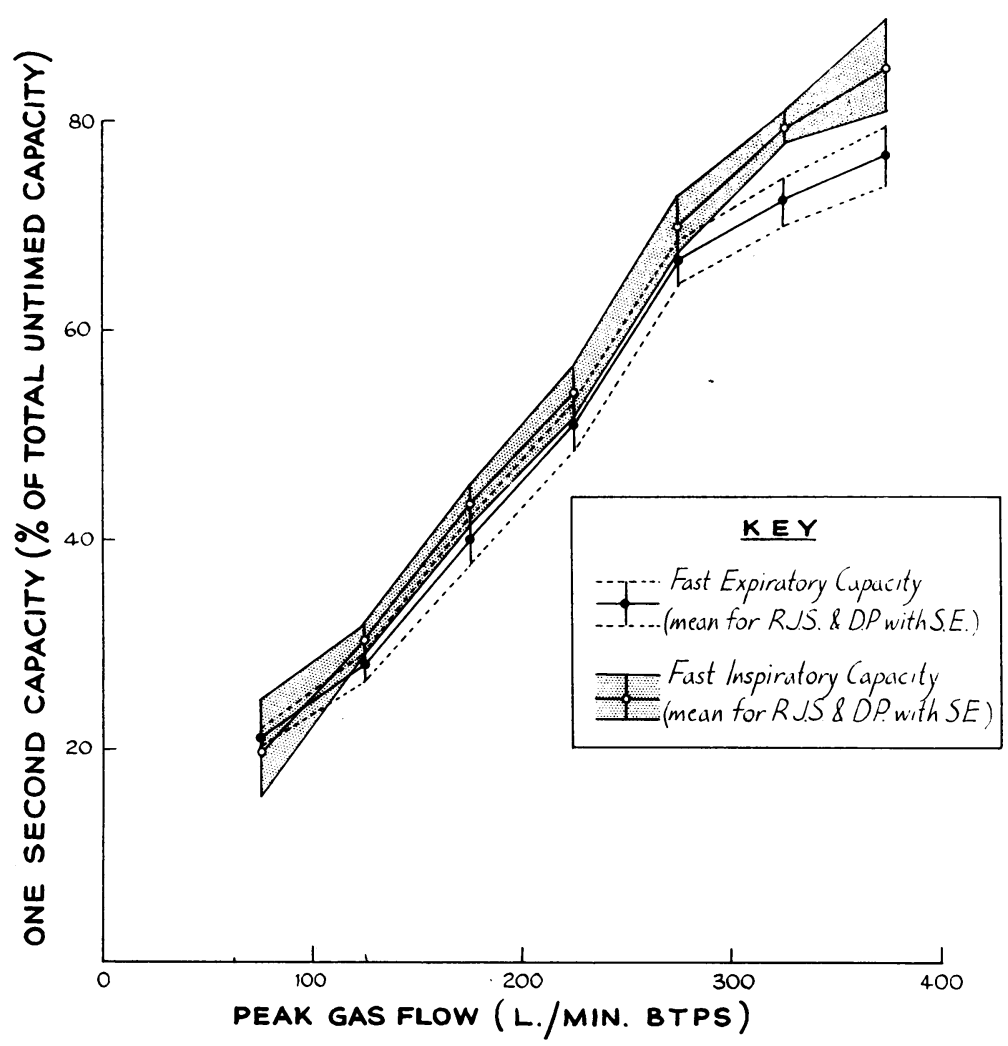

Fig. 6.-Relationship between peak rate of gas flow and magnitude of one-second capacity.

either time. The one-second capacity can be measured more accurately because the volume is greater and (if a spirometer is used) the oscillations are of smaller amplitude, so that there seems little advantage to be gained by the adoption of the shorter time interval.

Time Required to Reach a Peak Flow.-This gives some expression of the capacity of an individual to develop a high respiratory velocity from a given chest position (maximum inspiration or maximum expiration). The rate of increase of velocity in any one tracing is fairly constant, particularly at the higher velocities, and it is therefore possible to calculate an average value for the respiratory "acceleration" for the period during which respiratory velocity is increasing. There is a fairly wide individual variation in the magnitude of this acceleration, but both the principal subjects and five of the other six normal subjects develop a greater acceleration during expiration. It can be seen also that the acceleration decreases progressively as the total time taken over delivery is increased (Fig. 8).
Time Required for Gas Flow to Fall to a Low LEVEL.-The length of the " exponential tail" in individual tracings has been analysed by noting the time taken for the rate of flow to fall to a low level (30 1./min.). The difference between this time and the total time taken for delivery indicates the magnitude of the "tail." There is no significant difference between results for the two principal subjects, and both inspiratory and expiratory curves have a similar form (Fig. 9). As the delivery time is prolonged, the exponential tail increases in length, at first quite slowly, but if more than 6 seconds is taken all the additional time is added to the length of the "tail."

\section{Discussion}

In any procedure involving measurements over short periods of time it is desirable to use apparatus possessing a high natural frequency. However, until recently it was necessary to use a low frequency system (the spirometer) for the F.V.C. test, since no more suitable apparatus was available. The pneumotachograph provides a use- 
FIG. 7.-Relationship between peak rate of gas flow and time taken over delivery of gas sample. (Subject D. P.)

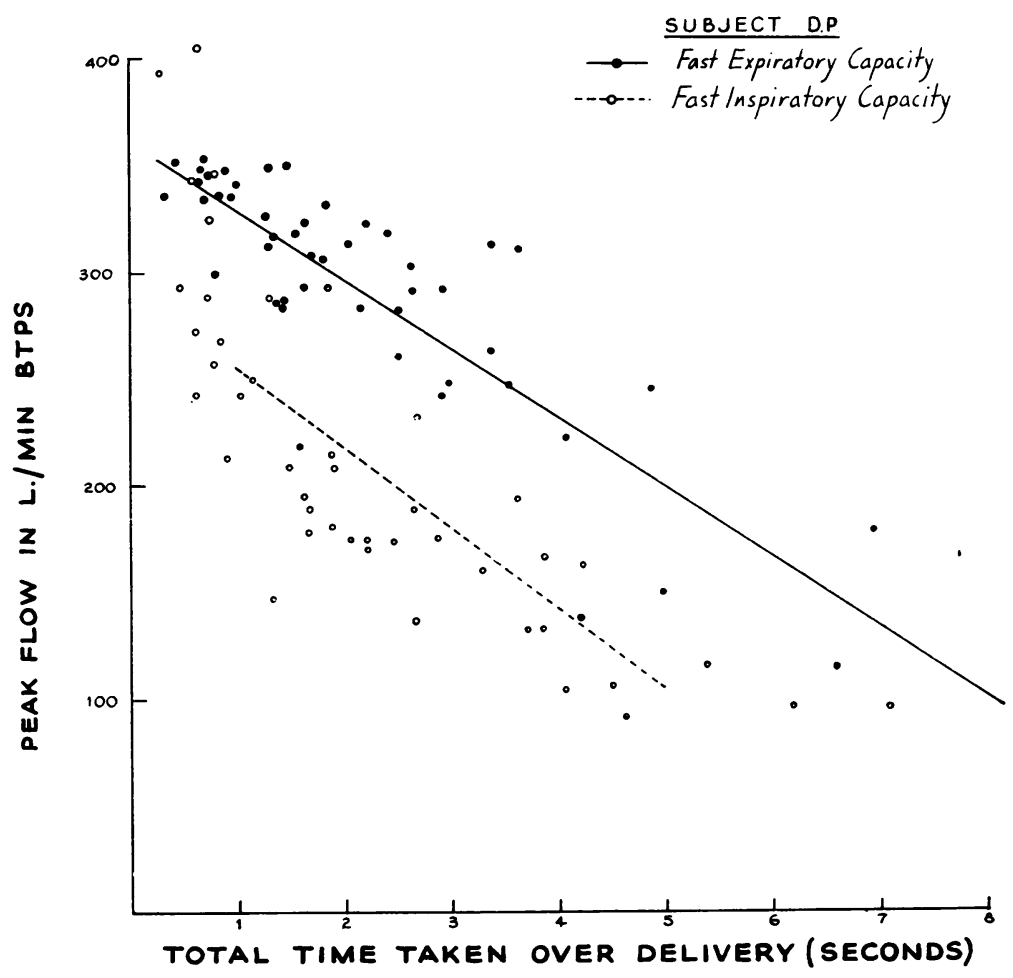

ful alternative apparatus with much higher frequency, and permits the investigation of a number of parameters of the F.V.C. It must now be considered how many of these have sufficient stability to be of clinical importance.

The conventional "one-second capacity" (Tiffeneau, Bousser, and Drutel, 1949 ; Roche and Thivollet, 1949 ; Gaensler, 1951 ; Hirdes and van Veen, 1952) is seriously influenced by the total time that the subject takes over the F.V.C. test ; many even of the normal subjects tend to take longer than the optimal time for a good result. However, this difficulty can be overcome either by relating the one-second capacity to total delivery time (as in Fig. 5), or by measuring the total fast capacity and relating this to delivery time (as in Fig. 4). The first procedure seems likely to be a more sensitive indicator of respiratory disability, although in normal subjects the second measurement shows a smaller variation, and is less affected by changes in the peak rate of gas flow. The peak gas flow also shows a fairly stable relationship with delivery time in a given subject, but the range of standardized values in different normal subjects is rather large, and clinical significance could be attached only to gross departures from normality. The same seems true of the onesecond flow-rate. The respiratory " acceleration" is a more promising measurement ; during inspiration in particular, the curve relating the acceleration to total delivery time shows a comparatively small variation. Finally, the length of the "exponential tail" seems a useful quantity. The variation in normal subjects is fairly small, and on general grounds it seems probable that the tail will be lengthened in conditions where the airway is narrowed.

In some laboratories it may still prove expedient to measure at least the one-second capacity by means of a spirometer. Where this is necessary, errors may be minimized by (i) a light-weight bell (Bernstein, D'Silva, and Mendel, 1952) to minimize the initial rise of pressure; (ii) a liquid of high viscosity, such as glycerol, in place of the water seal, to give increased damping of the oscillations that are induced; (iii) an approximate correction for the oscillations, based on a reconstruction of the actual wave form, as in Fig. 3. 


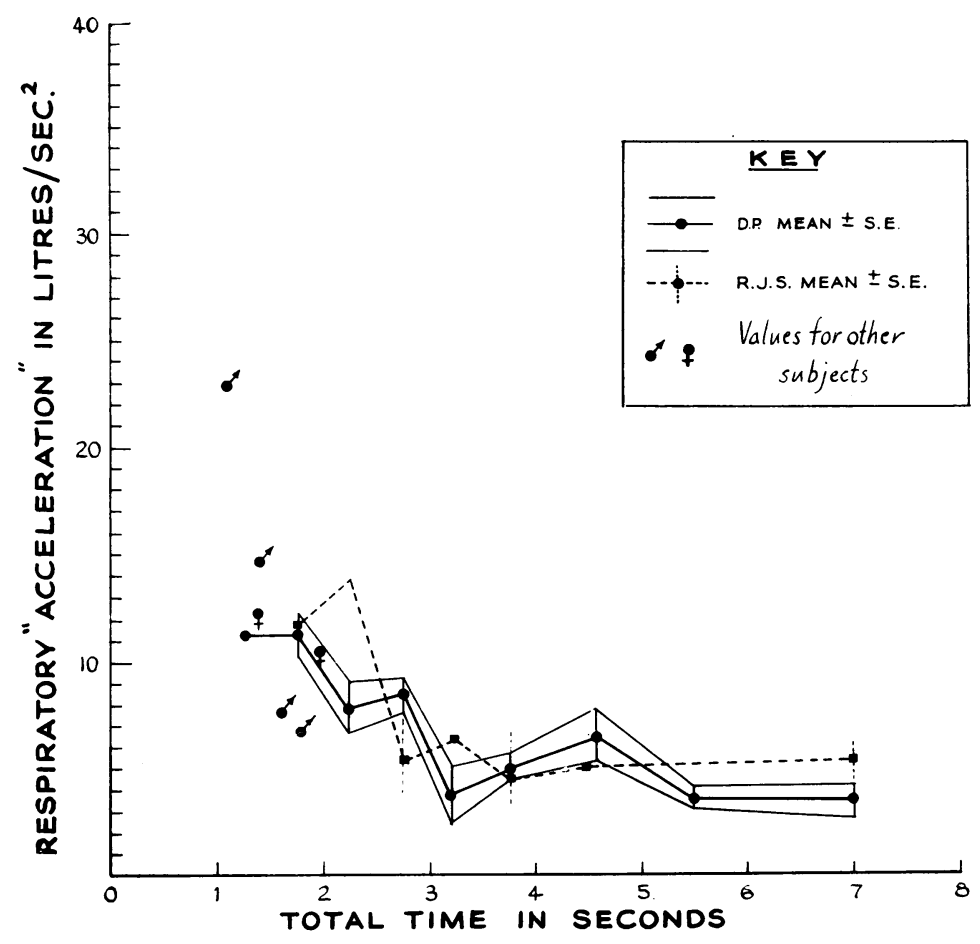

FIG. 8.-The average respiratory "acceleration" for the period during which respiratory velo-

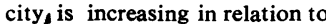
total time taken over delivery of sample. (a) I.F.V.C. (b) E.F.V.C.

FIG. $8 \mathrm{a}$

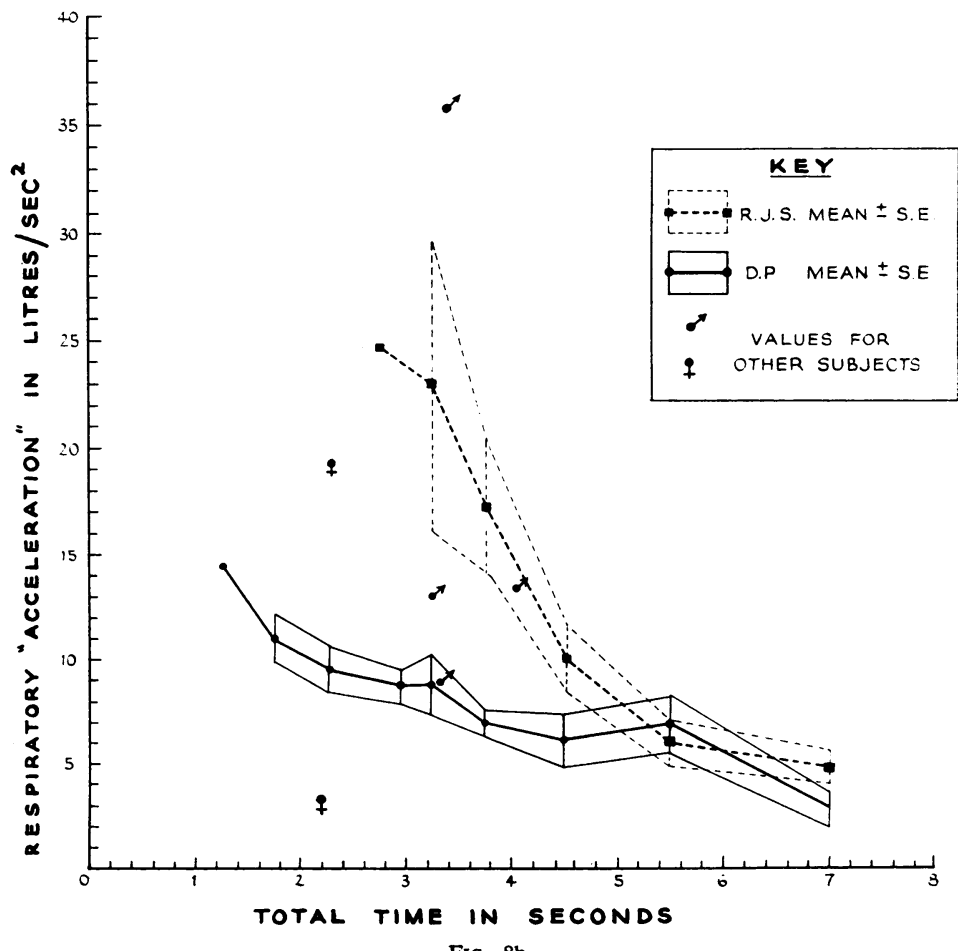

Fig. $8 b$ 


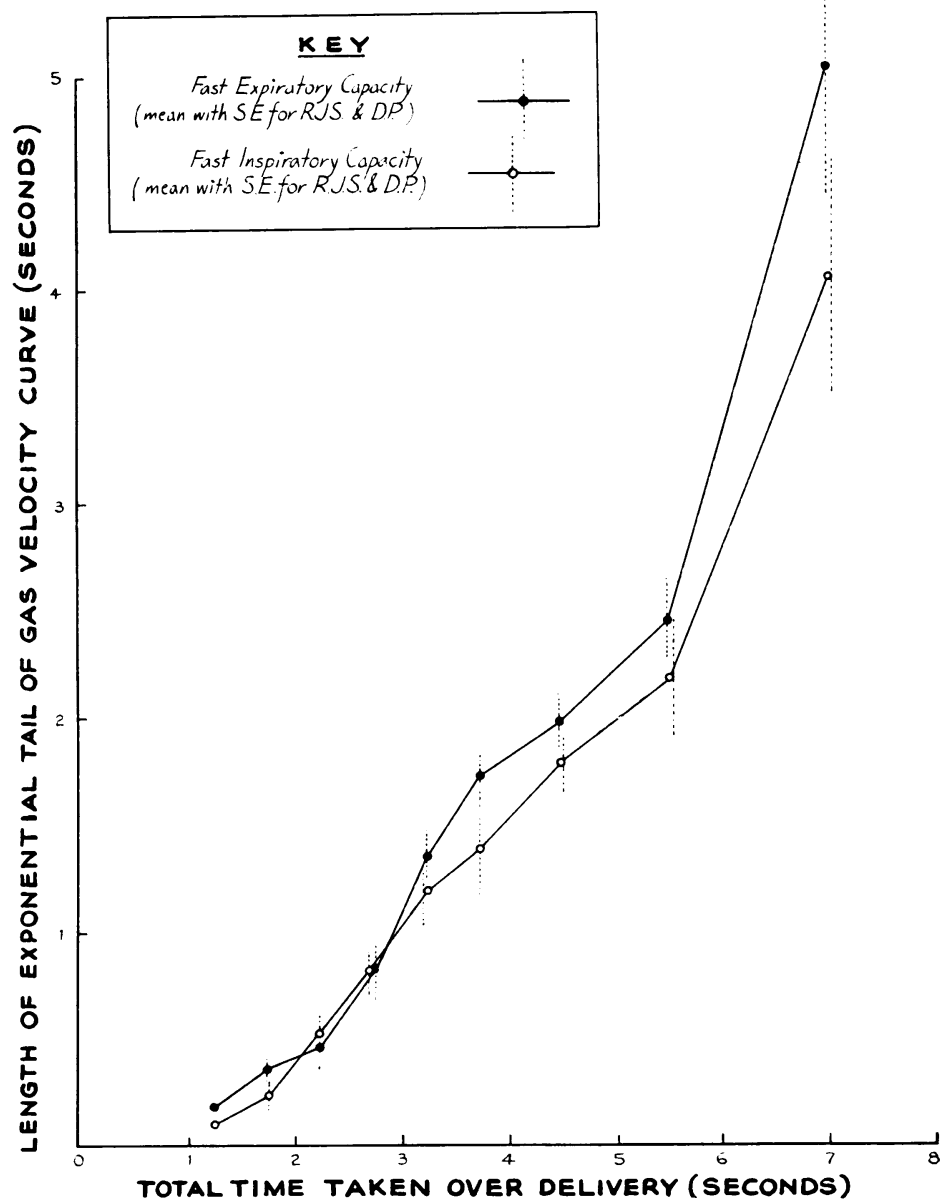

FIG. 9.-The length of the exponential tail of the gas flow curve (flow less than 30 $1 . / \mathrm{min}$.$) in relation to the total time taken over delivery of the gas sample.$

\section{SUMMARY}

Sources of error in the spirometer method of recording F.V.C. have been re-examined by simultaneous pneumotachograph and F.V.C. tracings, direct recording of pressure fluctuations in the spirometer bell, and detailed study of the wave form of oscillations induced under static and dynamic conditions. During the F.V.C. test, possible errors include (i) failure to indicate the true starting point of the test; (ii) the initiation of a more rapid respiratory acceleration than is found under conditions of minimal resistance ; and (iii) an obscuring of the end-point for the onesecond capacity due to oscillations of the spirometer bell.

The pneumotachograph avoids these difficulties, and permits a reasonably accurate measurement of a number of parameters of the F.V.C.
The one-second capacity still seems the most useful of these measures, but it must be assessed in relation to the total time taken over delivery of the sample. Other values that may prove of clinical interest are the respiratory acceleration and the length of the "exponential tail" of the velocity tracings.

\section{REFERENCES}

Bernstein, L. (1954). Thorax, 9, 63. and Kazantzis, G. (1954). Ibid., 9, 326

and Mendel, D. (1951). Ibid., 6, 297

- D'Silva, J. L., and Mendel, D. (1952). Ibid., 7, 255

Drutel, P., and Dechoux, J. (1952). J. franc. Méd. chir. thorac., 6, 517 Gaensler, E. A. (1951). Amer. Rev. Tuberc., 64, 256.

Gilson, J. C., and Hugh-Jones, P. (1949) Clin, Sci., 7, 185.

Germannsen, J. (1933). Z. ges. exp. Med., 90, 130.

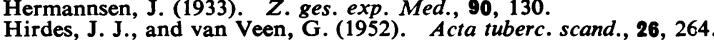
Hirdes, J. J., and van Veen, G. (1952). Acta

Needham, C. D., Rogan, M. C., and McDonald, I. (1955). Brit. J. Tuberc., 49, 30

Roche, L., and Thivollet, J. (1949). Arch. Mal. prof., 10, 448.

Shephard, R. J. (1955). Thorax, 10, 258.

Committee Report.

Simonin, P. (1954). Sem. Hôp. Paris, 30, 352. Tiffeneau, R.., Bousser, J., and Drutel, P. (1949). Paris méd. (partie
méd), 137, 543. 